\title{
Fuzzy random variables à la Kruse \& Meyer and à la Puri \& Ralescu: key differences and coincidences*
}

\author{
María Ángeles Gil
}

\begin{abstract}
The concept of the so-called fuzzy random variables has been introduced in the literature aiming to model random mechanisms 'producing' fuzzy values. However, the best known approaches (namely, the one by Kwakernaak-Kruse and Meyer and the one by Féron-Puri and Ralescu) have been thought to deal with two different situations and, to a great extent, with two different probabilistic and statistical targets. This contribution highlights some of the most remarkable differences and coincidences between the two approaches.
\end{abstract}

\section{Introduction}

Fuzzy sets were introduced by Zadeh in 1965 [18] to model classes of objects not having a precisely defined criterion of membership. In other words, a fuzzy set is a class for which elements from a referential classical set can be compatible to a lesser or greater extent with the (not necessarily well-defined) property characterizing such a class.

Since their introduction, fuzzy set and probability theories were connected, either to emphasize distinctions between the two types of underlying uncertainties, fuzziness $v s$ randomness (see Zadeh [18]) or to establish new settings and concepts involving/combining both (see, for instance, Zadeh [19]).

\footnotetext{
María Ángeles Gil

Universidad de Oviedo, Facultad de Ciencias, Departamento de Estadística, I.O. y D.M., Oviedo, Spain, e-mail: magil@uniovi.es

* The author is deeply indebted to the Editors of this book because of inviting her to contribute to this tribute to Professor Rudolf Kruse. I admire Kruse a lot, and as I was invited two decades ago by Roman Slowinski, Didier Dubois and Henri Prade to coauthor with him and Jörg Gebhardt a book chapter, it was a dream that came true. Since then, I have had chance to share meetings, conferences, scientific discussions, editing a special issue, and to have him invited to our university and to Asturias for different commitments. I am delighted for having now the opportunity of saying that all this has been a great privilege for me.
} 
In line with the second purpose, in 1976 Féron [6, 7] (see also [8]) introduced the notion of fuzzy random set to model a random mechanism 'producing' fuzzy values (more concretely, fuzzy sets of a metric space). Fuzzy random sets have been formalized either as random elements taking on values on spaces of fuzzy sets endowed with certain Borel $\sigma$-fields (i.e., following Fréchet's theory of random elements [9]) or, alternatively, as extending levelwise the notion of random sets. Féron's ideas were deeply considered and strengthened by Puri and Ralescu $[15,16]$ who re-coined fuzzy random sets as fuzzy random variables. Puri and Ralescu considered the specific metrics suggested by Fréchet and missing in Féron's papers, and they introduced key notions like expectation, conditional expectation, etc. In recent papers these random elements have been referred to as random fuzzy sets.

In accordance with the distinction epistemic/ontic of fuzzy values (see Couso and Dubois [4] for a recent review) Féron-Puri and Ralescu's concept corresponds to the ontic approach. Thus, fuzzy random variables in Féron-Puri and Ralescu's sense directly produce fuzzy-valued data. In fact, they are appropriate to model intrinsically imprecise-valued random attributes, like most of graded valuations associated with human ratings.

Almost simultaneously, in 1978 Kwakernaak $[13,14]$ introduced the notion of fuzzy random variable to formalize the fuzzy perception of an underlying realvalued random variable (called the original). In accordance with the distinction epistemic/ontic of fuzzy values, Kwakernaak's concept corresponds to the epistemic approach. Therefore, although the random mechanism behind fuzzy random variables in Kwakernaak's sense produce real-valued data, they cannot be/have not been exactly perceived but only a fuzzy perception of these data is available. Kwakernaak's ideas were formalized in a clearer mathematical way by Kruse [11] and Kruse and Meyer [12]. In summary, fuzzy random variables in Kwakernaak-Kruse-Meyer's sense are appropriate to model real-valued random attributes from which the available information is imprecise or is imprecisely reported.

This paper aims to review the key differences and analogies between these two approaches by recalling: their modelling, the way they address the formalization of the distribution and independence of fuzzy random variables, their main location and dispersion parameters, as well as a few comments about the statistical methods to analyze data from them.

\section{Two approaches to model fuzzy random variables}

In this section, the definitions for the two main approaches to random mechanisms producing fuzzy values are recalled. Both definitions have been stated in a probabilistic setting in which $(\Omega, \mathscr{A}, P)$ is the probability space modelling a random experiment, where $\Omega$ is the set of all possible experimental outcomes, $\mathscr{A}$ is a $\sigma$ field of subsets of $\Omega$ (the set of all possible events of interest), and $P$ is a probability measure associated with $(\Omega, \mathscr{A})$. 
Firstly, the revisitation of Kwakernaak's conceptualization by Kruse [11] and Kruse and Meyer [12] is given as follows:

Definition 1. Let $(\Omega, \mathscr{A}, P)$ be a probability space modelling a random experiment. Let $\mathscr{F}_{c}(\mathbb{R})$ be the space of all fuzzy numbers (i.e., piecewise continuous, normal and fuzzy convex sets of $\mathbb{R})$. A mapping $\mathscr{X}: \Omega \rightarrow \mathscr{F}_{c}(\mathbb{R})$ is said to be a fuzzy random variable $\grave{a} \boldsymbol{l} \boldsymbol{a}$ Kruse $\boldsymbol{\&}$ Meyer associated with $(\Omega, \mathscr{A}, P)$ if it satisfies for each $\alpha \in(0,1]$ that both $\inf \mathscr{X}_{\alpha}: \Omega \rightarrow \mathbb{R}$ and $\sup \mathscr{X}_{\alpha}: \Omega \rightarrow \mathbb{R}$ are real-valued random variables, where $\mathscr{X}_{\alpha}$ is the interval-valued $\alpha$-level mapping, $\mathscr{X}_{\alpha}(\omega)=(\mathscr{X}(\omega))_{\alpha}=\{x \in \mathbb{R}: \mathscr{X}(\omega)(x) \geq \alpha\}$, and inf $\mathscr{X}_{\alpha}(\omega), \sup \mathscr{X}_{\alpha}(\omega)$ $\in \mathscr{X}_{\alpha}(\omega)$, for all $\omega \in \Omega$.

Remark 1. Although it is not explicitly specified in the definition, Kwakernaak and Kruse and Meyer have clearly stated that a fuzzy random variable $\mathscr{X}: \Omega \rightarrow \mathscr{F}_{c}(\mathbb{R})$ in their sense is assumed to come from the composition of a real-valued random variable (the underlying one, referred to as the 'original', which is a mapping from $\Omega$ to $\mathbb{R}$ ) and a fuzzy perception (a mapping from $\mathbb{R}$ to $\mathscr{F}_{c}(\mathbb{R})$ ), that is,

$$
\begin{gathered}
\Omega \stackrel{\text { original random variable }}{\longrightarrow} \mathbb{R} \stackrel{\text { fuzzy perception }}{\longrightarrow} \mathscr{F}_{c}(\mathbb{R}) \\
\omega \mapsto[\text { fuzzy perception } \circ \text { original random variable }](\omega)=\mathscr{X}(\omega) .
\end{gathered}
$$

Secondly, the revisition of Féron's conceptualization by Puri and Ralescu $[15,16]$ is given as follows:

Definition 2. Let $(\Omega, \mathscr{A}, P)$ be a probability space modelling a random experiment. Let $\mathscr{F}\left(\mathbb{R}^{p}\right)$ be the class of fuzzy subsets $\widetilde{U}: \mathbb{R}^{p} \rightarrow[0,1]$ such that $\widetilde{U}_{\alpha}=\left\{x \in \mathbb{R}^{p}\right.$ : $\widetilde{U}(x) \geq \alpha\}$ is compact for each $\alpha \in(0,1]$ and $\widetilde{U}_{1} \neq \emptyset$. A fuzzy random variable $\grave{a} \mathbf{l a}$ Puri \& Ralescu associated with $(\Omega, \mathscr{A}, P)$ is a mapping $\mathscr{X}: \Omega \rightarrow \mathscr{F}\left(\mathbb{R}^{p}\right)$ such that for each $\alpha \in(0,1]$ the set-valued $\alpha$-level mapping $\mathscr{X}_{\alpha}$, with $\mathscr{X}_{\alpha}(\omega)=(\mathscr{X}(\omega))_{\alpha}$ for all $\omega \in \Omega$, is a random compact set (that is, a Borel-measurable mapping with respect to the Borel $\sigma$-field generated by the topology associated with the Haussdorf metric on the space of nonempty compact subsets of $\mathbb{R}^{p}$ ).

Remark 2. It should be emphasized that Colubi et al. [2, 3] have shown that fuzzy random variables à la Puri \& Ralescu are $\mathscr{F}\left(\mathbb{R}^{p}\right)$-valued random elements in Fréchet's sense, that is, they are Borel-measurable mappings w.r.t. the Borel $\sigma$-field generated by the topology associated with the Skorohod metric on $\mathscr{F}\left(\mathbb{R}^{p}\right)$. This Borel-measurability will be decisive for the ideas to be exposed in the next sections.

Remark 3. Although it is not explicitly specified in the definition, Féron and Puri and Ralescu have clearly stated that a fuzzy random variable $\mathscr{X}: \Omega \rightarrow \mathscr{F}_{c}(\mathbb{R})$ in their sense is assumed to come from the direct assessment of a fuzzy value to each experimental outcome, that is, 


$$
\begin{gathered}
\Omega \underset{\text { fuzzy-valued random element }}{\longrightarrow} \mathscr{F}_{c}(\mathbb{R}) \\
\omega \mapsto[\text { fuzzy-valued random element }](\omega)=\mathscr{X}(\omega) .
\end{gathered}
$$

As a conclusion from Remarks 1 and 3, the essential difference between the two approaches in defining fuzzy random variables (appart from the dimension of the Euclidean space fuzzy values are supposed to be defined on, and the fuzzy convexity of these values) lies in the situations they model. So, fuzzy random variables $a$ la Kruse \& Meyer assume the existence of a real-valued random process which is fuzzily perceived (epistemic view), whereas fuzzy random variables à la Puri \& Ralescu assume the existence of an imprecisely-valued random process for which imprecision is formalized in terms of fuzzy values (ontic view).

For some theoretical and most of practical developements, Definition 2 is particularized to the one-dimensional case $(p=1)$ and also to the fuzzy convex case (i.e., $\alpha$-levels are assumed to be convex sets). Under these particularizations the two definitions match, so that the essential coincidence between the two approaches in defining fuzzy random variables can be stated (see, for instance, Blanco-Fernández et al. [1] for a recent review about) as follows:

Proposition 1. Let $(\Omega, \mathscr{A}, P)$ be a probability space modelling a random experiment and let $\mathscr{F}_{c}(\mathbb{R})$ be the space of all fuzzy numbers. Then, a mapping $\mathscr{X}: \Omega$ $\rightarrow \mathscr{F}_{c}(\mathbb{R})$ is a fuzzy random variable à la Kruse \& Meyer if, and only if, it is a fuzzy random variable à la Puri \& Ralescu.

\section{Distribution and independence of fuzzy random variables}

As we have remarked in the preceding sections, the situations the two approaches to fuzzy random variables have been motivated on are different. This fact is crucial to support a key concept in dealing with probabilistic and statistical developments involving fuzzy random variables: the distribution and the independence.

If $\mathscr{X}$ is a fuzzy random variable à la Kruse $\&$ Meyer, its distribution is considered to be propagated from the distribution of the original through the fuzzy perception. This propagation is carried out on the basis of Zadeh's extension principle [20], so that the (fuzzy-valued) distribution function of fuzzy random variable $\mathscr{X}$ associated with $(\Omega, \mathscr{A}, P)$ is defined as follows:

$$
\begin{gathered}
\widetilde{F}_{\mathscr{X}}: \mathbb{R} \rightarrow \mathscr{F}_{c}(\mathbb{R}), \quad x \mapsto \widetilde{F}_{\mathscr{X}}(x): \mathbb{R} \rightarrow[0,1] \\
\left(\widetilde{F}_{\mathscr{X}}(x)\right)(p)= \begin{cases}\sup _{X_{0} \in \operatorname{Orig}(\mathscr{X}): F_{X_{0}}(x)=p} \inf _{\omega \in \Omega} \mathscr{X}(\omega)\left(X_{0}(\omega)\right) & \text { if } p \in[0,1] \\
0 & \text { otherwise }\end{cases}
\end{gathered}
$$

where $\operatorname{Orig}(\mathscr{X})$ is the set of potential originals of $\mathscr{X}$ and $F_{X}$ denotes the distribution function of random variable $X$ associated with $(\Omega, \mathscr{A}, P)$. 
As a consequence from this notion, two fuzzy random variables $\mathscr{X}$ and $\mathscr{Y}$ à $l a$ Kruse \& Meyer are defined to be identically distributed fuzzy random variables if their fuzzy distribution functions $\widetilde{F}_{\mathscr{X}}$ and $\widetilde{F}_{\mathscr{Y}}$ coincide for all $x \in \mathbb{R}$. This has been shown (see Kruse and Meyer [12]) to be equivalent to say that $\mathscr{X}$ and $\mathscr{Y}$ are identically distributed fuzzy random variables if and only if for each $\alpha \in(0,1]$ the random variables inf $\mathscr{X}_{\alpha}$ and $\inf \mathscr{Y}_{\alpha}$ are identically distributed and sup $\mathscr{X}_{\alpha}$ and $\sup \mathscr{Y}_{\alpha}$ are also identically distributed.

In a similar way, fuzzy random variables $\mathscr{X}_{1}, \ldots, \mathscr{X}_{n}$ à la Kruse $\&$ Meyer are defined to be (either pairwise or completely) independent fuzzy random variables if their joint fuzzy distribution functions can be (pairwise or completely) factorized in terms of the marginals. This has been shown (see Kruse and Meyer [12]) to be equivalent to say that $\mathscr{X}_{1}, \ldots, \mathscr{X}_{n}$ are (pairwise or completely) independent fuzzy random variables if and only if for each $\alpha \in(0,1]$ random variables $\inf \left(\mathscr{X}_{1}\right)_{\alpha}, \ldots, \inf \left(\mathscr{X}_{n}\right)_{\alpha}$ are (respectively, pairwise or completely) independent and $\sup \left(\mathscr{X}_{1}\right)_{\alpha}, \ldots, \sup \left(\mathscr{X}_{n}\right)_{\alpha}$ are also (respectively, pairwise or completely) independent.

On the other hand, if $\mathscr{X}$ is a fuzzy random variable $\grave{a}$ la Puri \& Ralescu, its distribution function cannot be directly extended, due to the lack of a universally accepted total order on the space of fuzzy values. However, because of the Borelmeasurability of a fuzzy random variable à la Puri \& Ralescu (Remark 2), one can immediately induce the distribution of this random element from the probability measure $P$ in $(\Omega, \mathscr{A}, P)$, so that for any Borel set $\mathfrak{B}$ of fuzzy values in $\mathscr{F}\left(\mathbb{R}^{p}\right)$, the (real-valued) induced probability of $\mathfrak{B}$ by $\mathscr{X}$ is given (without need to be specifically defined) by

$$
P(\mathscr{X} \in \mathfrak{B})=P(\{\omega \in \Omega: \mathscr{X}(\omega) \in \mathfrak{B}\}) .
$$

Analogously, the notions of identity in distribution and independence can be immediately derived on the basis of the Borel-measurability assumption for fuzzy random variables à $l a$ Puri \& Ralescu. Therefore, without need to be specifically defined, so that two fuzzy random variables $\mathscr{X}$ and $\mathscr{Y}$ à la Puri \& Ralescu are identically distributed fuzzy random variables if and only if for any Borel set $\mathfrak{B}$ of fuzzy values $P(\mathscr{X} \in \mathfrak{B})=P(\mathscr{Y} \in \mathfrak{B})$.

And, fuzzy random variables $\mathscr{X}_{1}, \ldots, \mathscr{X}_{n}$ à la Puri \& Ralescu are (either pairwise or completely) independent fuzzy random variables if for Borel sets $\mathfrak{B}_{1}, \ldots$, $\mathfrak{B}_{n}$ of fuzzy values

$$
\begin{gathered}
P\left(\mathscr{X}_{i} \in \mathfrak{B}_{i}, \mathscr{X}_{j} \in \mathfrak{B}_{j}\right)=P\left(\mathscr{X}_{i} \in \mathfrak{B}_{i}\right) \cdot P\left(\mathscr{X}_{j} \in \mathfrak{B}_{j}\right) \quad \text { for } i \neq j, \\
P\left(\mathscr{X}_{1} \in \mathfrak{B}_{1}, \ldots, \mathscr{X}_{n} \in \mathfrak{B}_{n}\right)=P\left(\mathscr{X}_{1} \in \mathfrak{B}_{1}\right) \cdot \ldots \cdot P\left(\mathscr{X}_{n} \in \mathfrak{B}_{n}\right) .
\end{gathered}
$$

Although the way to formalize the distribution of fuzzy random variables depends on the considered approach, so there is an essential difference in managing the distribution, the identity in distribution and independence in Puri \& Ralescu's approach imply those in Kruse \& Meyer's one in case one deals with fuzzy numbervalued random variables. 


\section{Parameters of the distribution of fuzzy random variables}

In summarizing the distribution of fuzzy random variables, the most used summary measures are location and dispersion ones. To extend them from the real-valued case the way to proceed depends on the approach and also on the way the distribution has been formalized.

In connection with the distribution of fuzzy random variables $\grave{a}$ la Kruse \& Meyer, extension is based on Zadeh's extension principle, so that a fuzzy parameter of a fuzzy random variable $\mathscr{X}$ is viewed as a fuzzy perception of a real-valued parameter of the original. Thus, if $\theta(X)$ denotes the parameter of the original $X$ the extended fuzzy perception of $\theta$ for $\mathscr{X}$ is given by the fuzzy number $\widetilde{\vartheta}(\mathscr{X})$ such that for each $t \in \mathbb{R}$

$$
\widetilde{\vartheta}(\mathscr{X})(t)=\sup _{X_{0} \in \operatorname{Orig}(\mathscr{X}): \theta\left(X_{0}\right)=t} \inf _{\omega \in \Omega} \mathscr{X}(\omega)\left(X_{0}(\omega)\right) .
$$

In accordance with this extension, if $\mathscr{X}$ is an $\grave{a}$ la Kruse $\&$ Meyer fuzzy random variable the main location measures are given by

- the fuzzy perception of the mean corresponds to

$$
\widetilde{E}(\mathscr{X})(t)=\sup _{X_{0} \in \operatorname{Orig}(\mathscr{X}): E\left(X_{0}\right)=t} \inf _{\omega \in \Omega} \mathscr{X}(\omega)\left(X_{0}(\omega)\right),
$$

which satisfies for each $\alpha \in(0,1]$ (see Kruse [11]) that

$$
(\widetilde{E}(\mathscr{X}))_{\alpha}=\left[E\left(\inf \mathscr{X}_{\alpha}\right), E\left(\sup \mathscr{X}_{\alpha}\right)\right] ;
$$

- the fuzzy perception of the median corresponds to

$$
\widetilde{\Gamma}(\mathscr{X})(t)=\sup _{X_{0} \in \operatorname{Orig}(\mathscr{X}): \operatorname{Me}\left(X_{0}\right)=t} \inf _{\omega \in \Omega} \mathscr{X}(\omega)\left(X_{0}(\omega)\right),
$$

which satisfies that for each $\alpha \in(0,1]$ (see Grzegorzewski [10])

$$
(\widetilde{\Gamma}(\mathscr{X}))_{\alpha}=\left[\underline{\operatorname{Me}}\left(\inf \mathscr{X}_{\alpha}\right), \overline{\operatorname{Me}}\left(\sup \mathscr{X}_{\alpha}\right)\right],
$$

where $\mathrm{Me} / \overline{\mathrm{Me}}$ denotes the median of the corresponding real-valued random variable with the convention (if the median is not unique) of taking the smallest/largest median.

In connection with the distribution of fuzzy random variables à la Puri \& Ralescu, extension is based on Fréchet's ideas [9] for random elements over metric spaces, so that if one considers the $L^{p}$ metrics $(p \in\{1,2\})$ by Diamond and Kloeden $[5]$ on $\mathscr{F}_{c}(\mathbb{R})$

$$
\rho_{p}(\widetilde{U}, \widetilde{V})=\left[\frac{1}{2} \int_{(0,1]}\left(\left|\inf \widetilde{U}_{\alpha}-\inf \widetilde{V}_{\alpha}\right|^{p}+\left|\sup \widetilde{U}_{\alpha}-\sup \widetilde{V}_{\alpha}\right|^{p}\right) d \alpha\right]^{1 / p} .
$$


In accordance with Fréchet's ideas, if $\mathscr{X}$ is an à la Puri \& Ralescu fuzzy random variable

- the extended mean corresponds to

$$
\widetilde{E}(\mathscr{X})=\arg \min _{\widetilde{U} \in \mathscr{F}\left(\mathbb{R}^{p}\right)} E\left(\left[\rho_{2}(\mathscr{X}, \widetilde{U})\right]^{2}\right),
$$

which satisfies for each $\alpha \in(0,1]$ (see Puri and Ralescu [16]) that $(\widetilde{E}(\mathscr{X}))_{\alpha}$ $=$ Aumann integral of $\mathscr{X}_{\alpha}$, and coincides with $\left[E\left(\inf \mathscr{X}_{\alpha}\right), E\left(\sup \mathscr{X}_{\alpha}\right)\right]$ if $\mathscr{X}_{\text {is }}$ $\mathscr{F}_{c}(\mathbb{R})$-valued;

- if $\mathscr{X}$ is $\mathscr{F}_{c}(\mathbb{R})$-valued, the extended (1-norm) median corresponds to

$$
\widetilde{\operatorname{Me}}(\mathscr{X})=\arg \min _{\widetilde{U} \in \mathscr{F}\left(\mathbb{R}^{p}\right)} E\left(\rho_{1}(\mathscr{X}, \widetilde{U})\right),
$$

for which a solution (see Sinova et al. [17]) is the one such that for each $\alpha \in(0,1]$ is given by $(\widetilde{\mathrm{Me}}(\mathscr{X}))_{\alpha}=\left[\mathrm{Me}\left(\inf \mathscr{X}_{\alpha}\right), \mathrm{Me}\left(\sup \mathscr{X}_{\alpha}\right)\right]$, where Me denotes the median of the corresponding real-valued random variable with the convention (if the median is not unique) of taking the middle median. Actually, some other conventions could be considered to choose Me (like the one leading to $\widetilde{\Gamma}(\mathscr{X})$ ), whenever a fuzzy number is determined.

Regarding the variance, the policy for this approach is essentially different from an approach to the other. If $\mathscr{X}$ is an $\grave{a}$ la Kruse $\&$ Meyer fuzzy random variable and Zadeh's extension principle is applied, the variance is conceived as a fuzzy perception of the variance of the original, so it is fuzzy-valued. If $\mathscr{X}$ is an à la Puri $\&$ Ralescu fuzzy random variable and Fréchet's ideas are applied, the variance is conceived as a real-valued measure given by the mean squared $L^{2}$ distance between the fuzzy random variable and its mean value. Consequently, they stand for two different types of measures.

\section{Statistical data analysis from fuzzy random variables}

The approach behind the two examined notions for fuzzy random variables influences the statistical data analysis one can develop. Since there is a wide class of methods to estimation and testing hypothesis from fuzzy data, the topic cannot be entered in this paper.

Anyway, it is interesting to highlight that analyses concerning fuzzy random variables $\grave{a}$ la Kruse \& Meyer can refer either to parameters of the originals or to fuzzy perceptions of them (see, for instance, Kruse and Meyer [12] for several examples). In contrast to this, analyses concerning fuzzy random variables à la Puri \& Ralescu always refer to parameters of the distribution of the fuzzy random variables (see for instance, Blanco-Fernández et al. [1]). Actually, in connection with the last ones, 
it should be pointed out that, thanks to having modelled fuzzy random variables as random elements, all the basic concepts from statistics with crisp data (e.g., unbiased estimation, $p$-values, and do on, etc.) can be preserved without needing to expressly define them.

Following Kruse, the analysis of fuzzy-valued data should be clearly distinguished from the analysis of data by using fuzzy logic-based methods.

Acknowledgements The research in this paper has been partially supported by the Principality of Asturias/FEDER Grant GRUPIN14-101 and the Spanish Ministry of Economy and Competitiveness Grant MTM2015-63971-P. Their financial support is gratefully acknowledged.

\section{References}

1. Blanco-Fernández, A., Casals, M.R., Colubi, A., Corral, N., García-Bárzana, M., Gil, M.A., González-Rodríguez, G., López, M.T., Lubiano, M.A., Montenegro, M., Ramos-Guajardo, A.B., de la Rosa de Sáa, S., Sinova, B.: A distance-based statistical analysis of fuzzy numbervalued data. Int. J. Approx. Reas. 55, 1487-1501 (2014)

2. Colubi, A., Domínguez-Menchero, J.S., López-Díaz, M., Ralescu, D.A.: On the formalization of fuzzy random variables. Inform. Sci. 133, 3-6 (2001)

3. Colubi, A., Domínguez-Menchero, J.S., López-Díaz, M., Ralescu, D.A.: A $D_{E}[0,1]$ representation of random upper semicontinuous functions. Proc. Amer. Math. Soc. 130, 3237-3242 (2002)

4. Couso, I., Dubois, D.: Statistical reasoning with set-valued information: Ontic vs. epistemic views. Int. J. Approx. Reas. 55 1502-1518 (2014)

5. Diamond, P., Kloeden, P.: Metric spaces of fuzzy sets. Fuzzy Sets Syst. 35, 241-249 (1990)

6. Féron, R.: Ensembles aléatoires flous. C. R. Acad. Sc. Paris A 282, 903-906 (1976)

7. Féron, R.: Ensembles flous attachés à un ensemble aléatoire flou. Publ. Econometr. 9, 51-66 (1976)

8. Féron, R.: Sur les notions de distance et d'ecart dans une structure floue et leurs applications aux ensembles aléatoires flous. C. R. Acad. Sc. Paris A 289, 35-38 (1979)

9. Fréchet, M.: Les éléments aléatoires de nature quelconque dans un espace distancié. Ann. L'Inst. H. Poincaré 10, 215-310 (1948)

10. Grzegorzewski, P.: Statistical inference about the median from vague data. Contr. Cyber. 27, 447-464 (1998)

11. Kruse, R.: The Strong Law of Large Numbers for fuzzy random variables. Inform. Sci. 28, 233-241 (1982)

12. Kruse, R., Meyer, K.D.: Statistics with Vague Data. Reidel, Dordrecht (1987)

13. Kwakernaak H.: Fuzzy Random Variables, Part I: Definitions and Theorems. Inform. Sci. 15, $1-15(1978)$

14. Kwakernaak H.: Fuzzy Random Variables, Part II: Algorithms and Examples for the Discrete Case. Inform. Sci. 17, 253-278 (1979)

15. Puri, M.L., Ralescu, D.A.: The concept of normality for fuzzy random variables. Ann. Probab., 11, 1373-1379 (1985)

16. Puri, M.L., Ralescu, D.A.: Fuzzy random variables. J. Math. Anal. Appl., 114, 409-422 (1986)

17. Sinova, B., Gil, M.A., Colubi, A., Van Aelst, S.: The median of a random fuzzy number. The 1-norm distance approach. Fuzzy Sets Syst. 200, 99-115 (2012)

18. Zadeh, L.A.: Fuzzy sets. Inform. Contr. 8, 338-353 (1965)

19. Zadeh, L.A.: Probability measures of Fuzzy events. J. Math. Anal. Appl. 23, 421-427 (1968)

20. Zadeh, L.A.: The concept of a linguistic variable and its application to approximate reasoning. Part 1: Inform. Sci. 8, 199-249; Part 2: Inform. Sci. 8, 301-353; Part 3: Inform. Sci. 9, 43-80 (1975) 